\begin{tabular}{|c|l|}
\hline Title & A comparison of intertemporal choices for oneself versus someone else based on Tsallis' statistics \\
\hline Author(s) & Takahashi, Taiki \\
\hline Citation & $\begin{array}{l}\text { Physica A: Statistical Mechanics and its A pplications, 385(2), 637-644 } \\
\text { https://doi.org/L0.1016/.physa.2007.07.020 }\end{array}$ \\
\hline Issue Date & 2007 \\
\hline Doc URL & http://hdl.handle.net/2115/50613 \\
\hline Type & article (author version) \\
\hline File Information & For Huscap Takahashi_PHYSICA_A_SelfOtherDiscounting.pdf \\
\hline
\end{tabular}

Instructions for use 
[This paper appeared in PHYSICA A: STATISTICAL MECHANICS AND ITS APPLICATIONS (Physica A 385 (2007) 637-644)]

\title{
A comparison of intertemporal choices for oneself versus someone else based on Tsallis' statistics.
}

\author{
Taiki Takahashi ${ }^{1}$
}

${ }^{1}$ Direct all correspondence to Taiki Takahashi, Unit of Cognitive and Behavioral Sciences, Department of Life Sciences, School of Arts and Sciences, The University of Tokyo, Komaba, Meguro-ku, Tokyo, 153-8902, Japan (taikitakahashi@gmail.com).

Acknowledgements: The research reported in this paper was supported by a grant from the Grant- in-Aid for Scientific Research ("21st century center of excellence" grant) from the Ministry of Education, Culture, Sports, Science and Technology of Japan. 


\section{Summary}

Impulsivity and inconsistency in intertemporal choice (discounting) have drawn attention in econophysics and neuroeconomics. Although it is well established that most people often show irrational discounting (e.g. hyperbolic discounting), little is known regarding whether the irrationality is mitigated or not, when the choice was performed by someone else instead of subject herself. This point is important for economic policy-making. In order to compare consistency and impulsivity in choices for oneself versus someone else, we experimentally estimated the consistency parameter $q$ in Tsallis' statistics-based discount function for oneself and someone else, by assessing the points of subjective equality (indifference points) at seven delays (one week-25 years) in humans. We observed that (i) most people are more inconsistent when the outcomes of intertemporal choice are only relevant to someone else $(q=-8.89)$ than when relevant to oneself $(q=-2.63)$, and (ii) impulsivity, distinguished from inconsistency by utilizing the Tsallis statistics-based q-exponential discount function, is also larger in the choice for someone else than for oneself. Our results indicate that (i) leaving decision-making processes with some others may neither reduce impulsivity nor correct inconsistency, and (ii) when q-exponential discount is utilized, the definition range of $q$-parameter should be extended to $q<0$, and smaller $q<1$ and larger $q>1$ indicate more inconsistent discounting. Together, the usefulness of the q-exponential discounting for analyzing the dynamic consistency of economic policy was demonstrated in the present study.

Keywords: Econophysics; Intertemporal choice; Inconsistency; Neuroeconomics; Discounting 


\section{Introduction to intertemporal choice behavior}

In intertemporal choice, people usually prefer an immediate reward to a delayed reward (delay discounting) [1-7]. Impulsivity in intertemporal choice refers to marked preference for smaller but more immediate over larger but more delayed rewards. "Impulsivity" here is a term often utilized in psychiatric studies on intertemporal choice---impulsive subjects such as smokers, addicts and attention deficit hyperactivity-disorder patients are unable to wait for delayed reward, resulting in strong preference for sooner smaller rewards over later larger rewards [1,3,4,7]. For instance, heroin addicts prefer "sooner but (objectively) smaller" rewards (i.e., pleasures from drug intake) over "later but (objectively) larger" rewards (e.g., a long lifespan, and a healthy and rich elderly life). This preference for sooner rewards (subjective devaluation of objectively large but delayed reward) per se is, theoretically speaking, neither irrational nor paradoxical, since there may be logical reasons for the discounting of a delayed reward; e.g., uncertainty of delayed rewards and an opportunity cost of delaying the acquisition of rewards.

First, suppose the following example 1 for demonstrating impulsivity and patience. Agent A who prefers "one orange available one year later" over "two oranges available [one year plus one week] later" is more impulsive than agent B who prefers "two oranges available [one year plus one week] later" over "one orange available one year later". In this example 1 , most people may behave like the patient agent B. It is to be noted that both impulsive agent $\mathrm{A}$ and patient agent $\mathrm{B}$ may be rational, because, in this example 1 alone, there is no inconsistency even in impulsive agent A's behavior. What types of psychological processing mediates temporal discounting and its relation to uncertainty aversion is under extensive investigations [2-3].

There is, however, a paradoxical phenomenon. Suppose the next intertemporal choice example 2. There are two options: "one orange available today" and "two oranges available one week later". In example 2, most people (who planned to choose the later option in example 1) simultaneously tend to prefer "one orange available today" over "two oranges available one week later" (it is known a small number of people may prefer the later option in example 2, but almost no one prefers the sooner option in example 1) [1,2]. Although the single choice of the sooner reward in the example 2 alone is not irrational, the combination (or equivalently, the co-occurrence within the same single subject) of these two intertemproal choices in example 1 (choosing the later) and example 2 (choosing the sooner) is inconsistent. The reason is that the time-intervals between sooner and delayed rewards are the same (i.e., 7 days) in the two intertemporal choice problems. Most people prefer later rewards in the distant 
future (example 1) but prefer sooner rewards when the times of receiving the two delayed rewards approaches to the near future (example 2), even if the time-interval between the two options were unchanged.

This inconsistency in inetertemporal choice behavior leads to the reversal of planned choice in example 1, at the time point of example 2 (one-year after the decision-making in example 1), solely due to the passage of time. In physical terms, there is a breaking of time-translational symmetry in human intertemporal choice. Note that a time-decay dynamics of radioactivity of plutonium etc. has this symmetry because the decay-rate of the radioactive isotope is constant over time; on the contrary, the "decay rate" of the subjective value of the delayed rewards may be a decreasing function of delay, and the orders of subjective values (preferences) of two delayed rewards will be changed as time passes. Psychologically speaking, most subjects may overestimate their patience at the distant future.

In order to specify the terms "impulsivity" and "inconsistency" here, let us further consider the following behaviors in the example 1 and 2: if agent A in example 1 (preferred the sooner: "one orange available one year later") again prefers the sooner option in example 2 ("one orange available today"), she is not inconsistent (this agent is consistent and impulsive), and if agent B (preferred the later option in example 1) again prefers the later option: "two oranges available one week later" in example 2, she is consistent and patient (both agents $\mathrm{A}$ and $\mathrm{B}$ are perfectly rational in these cases, unlike most people). In summary, if agents prefer larger later rewards in the distant future, but prefer smaller sooner rewards in the near future, their intertemproal choices are dynamically inconsistent, because their preferences reverse as time passes [1-4]. It is to be stressed here that example 1 is about a plan regarding intertemporal choice in the distant future at one year later from now (because the agents cannot take future actions now); while example 2 is about an intertemporal choice action.

The introduced widely-observed inconsistency between intertemporal choice plans and actions is problematic in that even if the agent had made patient and forward-looking plans about the distant future (as in example 1), her choice plan will, as the time of executing the plan approaches to the present, be canceled and more impulsive choice will instead be taken at the moment of intertemporal choice actions, as shown in example 2 (referred to as "preference reversal" [1-3]). This indicates that there is a discrepancy between the decision-maker's intention (will) and action; in other words, most people cannot act as they intended/planned in advance, in intertemporal choice problems [1]. Economic studies have demonstrated that this inconsistency may explain various problematic behaviors such as loss of self-control, a failure in formerly-planned 
abstinence from addictive substances and relapse, a dead-line rush due to procrastination, and a failure in saving enough before retirement (although people at younger ages tend to plan/want to save enough by the time of retirement in later life, most of them at middle ages do not reduce consumptions enough---a typical example of discrepancy between a plan/intention and an action in intertemporal choice) [1-3]. Recent neuroeconomic studies have demonstrated that intertemporal choice behavior is modulated by neurochemical substrates such as nicotine, cortisol, and testosterone [4-6]. Moreover, dynamic inconsistency is problematic not only for individuals but also for governmental economic policies [8].

Dynamically consistent (rational) temporal discounting (exponential discounting), which has been assumed in classical economic theory, follows the exponential equation [1-7]:

$$
V(D)=A \exp \left(-k_{e} D\right)
$$

(Equation 1)

where $\mathrm{V}$ is the subjective value of a reward, $\mathrm{A}$ is the subjective value of an immediate reward, and $\mathrm{D}$ is the length of delay until the delivery of reward. The free parameter $k_{e}$ is an index of the degree to which a subject discounts the delayed reward, i.e., larger $k_{e}$ values correspond to steeper delay discounting. In exponential discounting, there is no dynamic inconsistency. However, as introduced above, human (and animal) subjects often display preference reversals as time passes [1-4], because the following hyperbolic discount equation significantly fits behavioral data (better than the exponential discounting):

$$
V(D)=A /\left(1+k_{h} D\right) \text {. }
$$

(Equation 2)

where large $k_{h}$ values again correspond to steep discounting. It is important to note that, in exponential discounting, a discount rate $=-(d V / d D) / V=k_{e}$ (independent of delay) is constant over time. In contrast, in hyperbolic discounting, the discounting rate = $-(d V / d D) / V=k_{h} /\left(1+k_{h} D\right)$ (a hyperbolic discount rate) is a decreasing function of delay ("increasing patience" or "decreasing impatience"), resulting in preference reversal as time passes (i.e., hyperbolic discounter's plan at $D>0$ is patient but her action at $D=0$ is impulsive) [1-4]. In order to quantify the two distinct type of irrational/problematic tendencies in intertemporal choice (i.e., inconsistency and impulsivity), recent studies in econophysics and neuroeconomics [9] have introduced a q-exponential discount function based on Tsallis' statistics [10-11] which will be addressed in the next section. 


\section{Discount function based on Tsallis' statistics}

A recent study on intertemporal choice [10] proposed the q-exponential (i.e., $\left.\exp _{q}(x):=[1+(1-q) x] 1 /[1-q]\right)$ discount function:

$$
V_{q}(D)=A / \exp _{q}\left(k_{q} D\right)=A /\left[1+(1-q) k_{q} D\right]^{1 /(1-q)} \quad \text { (Equation 3) }
$$

where $A$ is the objective amount of a reward $\left(=V_{q}(0)\right)$ and $k_{q}$ is a parameter of impulsivity at delay $D=0$ (q-exponential discount rate at delay $\mathrm{D}=0$ ). Note that when $q=0$, equation 3 is the same as a hyperbolic discount function (equation 2), while $q \rightarrow 1$, is the same as an exponential discount function (equation 1). We have previously demonstrated that this q-parameter can be used to assess a subject's consistency in intertemporal choice in behavioral studies ( $q=0$ corresponds to hyperbolic discounting; while $\mathrm{q}=1$, complete consistency, corresponds to exponential discounting) and observed that most people have inconsistent intertemporal choice behavior when the outcomes were relevant to decision-makers themselves [11].

For precisely interpreting behavioral data in intertemporal choice, it is now important to examine the dependency of the q-exponential discount rate (qEDR) on $q$ and delay D. We can see

$$
\begin{aligned}
\mathrm{qEDR} & :=-\left(d V_{q} / d D\right) / V_{q} \\
& =k_{q} /\left(1+k_{q}(1-q) D\right) .
\end{aligned}
$$

Therefore, $\mathrm{qEDR}=k_{q}$ at delay $\mathrm{D}=0$, for any $\mathrm{q}$. It is to be noted that the impulsivity of actual intertemporal behavior action is parametrized with $k_{q}$, because actual actions occur at delay $\mathrm{D}=0$ (not at $\mathrm{D}>0$ ). On the other hand, the value of $\mathrm{qEDR}$ at delay $\mathrm{D}>0$ indicates the impulsivity in the plan of future intertemporal choice behavior, because humans cannot take future actions at delay $\mathrm{D}>0$ at the present time (delay $\mathrm{D}=0$ ). Further, it is critical to examine how the inconsistency (time-dependency of qEDR) depends on the parameters in the q-exponential discount model. The time-dependency of qEDR is expressed as:

$$
(d / d D)(\mathrm{qEDR})=-k_{q}^{2}(1-q) /\left(k_{q}(1-q) D+1\right)^{2} .
$$

From Equation 5, we can see the following three cases according to q: 
Case (a): $(d / d D)(\mathrm{qEDR})<0$ for $\mathrm{q}<1$ (decreasing impulsivity),

Case (b): $(d / d D)(\mathrm{qEDR})=0$ for $\mathrm{q}=1$ (exponential discounting, consistent intertemporal choice),

Case (c): $(d / d D)(\mathrm{qEDR})>0$ for $\mathrm{q}>1$ (increasing impulsivity).

It should now be noticed that hyperbolic discounting is a special case of (a) (i.e., q=0) and most people, who have decreasing impulsivity, are in case (a), few completely rational decision-makers are in case (b), and almost no one may be in case (c), according to the introduced empirical studies [1-3]. This analysis shows that, although an original proposal of the q-exponential discount model assumed that $\mathrm{q}$ is non-negative [10], the q-exponential discount model is well-defined also for $\mathrm{q}<0$.

Let us further examine the property of the absolute value of $[(d / d D)(\mathrm{qEDR})$ at $\mathrm{D}=0]=\left|k_{q}^{2}(q-1)\right|$. This quantity indicates the degree of inconsistency at the time of the intertemporal choice action occurring at $\mathrm{D}=0$ (a discrepancy between plan at $\mathrm{D}>0$ and action at $\mathrm{D}=0)$. Because this is an increasing function of $|q-1|$, inconsistency of the action increases as q deviates from 1 . Therefore, for $\mathrm{q}<1$, smaller $\mathrm{q}$ values indicate more inconsistent intertemporal choice (more rapidly decreasing impulsivity at $D=0$ for smaller $\mathrm{q}<1$ ); on the contrary, for $\mathrm{q}>1$, larger $\mathrm{q}$ values indicate more inconsistent intertemporal choice (more rapidly increasing impulsivity at $D=0$ for larger $q>1$ ). Consequently, negative q values correspond to more rapidly decreasing impulsivity compared to a conventional hyperbolic discounter's impulsivity as a function of delay ("more hyperbolic" discounting). This type of extremely inconsistent discount functions has never been proposed until the q-exponential discount function was introduced by Cajueiro [10] and analyzed here (a similar type function, a general hyperbola has been proposed by several authors in discounting literature [2], but the general hyperbola has never been examined in terms of consistency before Cajueiro's study [10]). Notably, smaller q values imply more inconsistent intertemporal choice for the domain $-\infty<q<1$ in which most people may be included. In summary, at $\mathrm{D}=0$ and for $q \leq 1$ (most people's $q$ satisfies this inequality [11]), higher degrees of impulsivity and inconsistency are characterized by larger $k_{q}$ and smaller $q$, respectively.

It can be concluded from these analysis, that if the agent has a large $k_{q}$ and small $q$ values ( $\mathrm{q}<1$ ), the agent's intertemporal choice plan (at delay $\mathrm{D}>0$ ) is patient but her intertemporal choice action (at delay $\mathrm{D}=0$ ) is impulsive; in other words, the effect of dynamic inconsistency (a discrepancy between plans and actions) is stronger for larger $k_{q}$ and smaller $q$. Importantly, this type of discounting behavior will, incorrectly, be considered patient, in both hyperbolic and exponential discount models, because these 
models do not have the inconsistency parameter and the estimated values of $k_{h}$ and $k_{e}$ will be small for this type of intertemporal choice. Namely, smaller $k_{h}$ and $k_{e}$ do not always indicate patience in actual intertemporal choice actions at delay $\mathrm{D}=0$, but only indicate patience in plans in the future intertemporal choice (at delay $\mathrm{D}>0$ ). This finding indicates the advantage of the q-exponential discount model for quantifying agent's impulsivity in actual intertemporal choice actions.

Together, it is important to examine the degree of inconsistency in intertemporal choice when the outcomes are only relevant to other people (i.e., irrelevant to decision-makers themselves) for the establishment of more efficient, consistent and beneficial economic policy-making. However, to date, no study to our knowledge has actually compared consistencies (and impulsivities) in intertemporal choices for oneself versus someone else.

\section{Behavioral data}

We conducted a behavioral experiment assessing subjects' discount behavior relevant to (a) subjects themselves and (b) other people (irrelevant to decision-making subject), by adopting a similar procedure of previous studies [4,12] (briefly, $A=1,000$ yen, with 7 delays ranging from 1 week to 25 years). Subjects were graduate students of the University of Tokyo $(\mathrm{N}=20)$. The experimental procedures determined participant's discounted presented values (indifferent points) at the seven delays. The total experimental procedure took a participant about 1 hour to conduct. The sample size is standard in behavioral economic studies on intertemporal choice [2,4,5,11,12]. All participants performed the two types of intertemproal choice tasks; i.e., the reward is for oneself and for someone else (the experiment was a within-subject design). In intertemporal choice for oneself, participants are informed that [the options are about money you will receive either immediately or later. Suppose as if the options were about real money]. In the task of intertemporal choice for someone else, participants were instructed as [the options are about money which someone else will receive either immediately or later. Suppose that you do not have to pay the money; the money will be paid from a fund sacred to charity or tax revenue. Please select options between immediate and delayed monetary endowment for the person. In making your decisions, suppose as if the options were about real money].

After conducting the experiment, we plotted the group median of the assessed discounted present value (indifference point) for each delay, in intertemporal choice for oneself and someone else (see Figure 1), following previous studies [2, 12]. We then 
conducted nonlinear curve fitting by utilizing the Gauss-Newton method implemented in R statistical language (http://www.r-project.org/), in order to estimate parameters in the discount models (Table 1). We fitted the q-exponential, exponential and hyperbolic models to the group median data. The goodness-of-fit was evaluated with AIC (Akaike Information Criterion):=2[(the number of parameters)-(logged likelihood)], because the number of parameters in the q-discount model is larger than those of two other models and the relation between the delay and the discounted presented value is non-linear. Note that smaller AICs indicate better fitting, as can be seen from the definition of AIC above.

Regarding the goodness-of-fit, in intertemporal choice for oneself, AICs were $80.06<87.36<92.72$ for the q-exponential, hyperbolic and exponential models, respectively. Likewise, for intertemporal choice for someone else, AICs were $66.84<96.91<99.56$, for the q-exponential, hyperbolic and exponential models, respectively. These results of AIC indicates that the order of goodness-of-fit was [q-exponential model>hyperbolic model>exponential model] for both self-relevant and -irelevant intertemporal choices. The results demonstrate the advantage of the q-exponential discount model. The better fitting of hyperbolic than exponential discounting is in line with a number of previous reports that human intertemporal choice is inconsistent [1-3]. Further, as will be discussed later, the estimated q values were negative (Table 1), indicating that participants typical intertemporal choice was "more hyperbolic"; i.e., more inconsistent discounting than hyperbolic discounting (corresponding to $\mathrm{q}=0$ in the $\mathrm{q}$-discount model). This observation has been impossible until Tsallis' statistics-based q-exponential discount model was introduced to the research domain of discounting behavior [10,11]. Fitted curves of the q-exponential, hyperbolic, and exponential models for both oneself and someone else are presented in Figure 2 and will more extensively be discussed below.

We can see in Fig. 1 that intertemporal choice for someone else appears to be less impulsive especially in the distant future (i.e., the discounted present values of money delayed more than 1 year for someone else are larger than those for oneself). However, this does not necessarily indicate that leaving intertemporal decision-making processes with some other persons leads to more rational and patient intertemporal choice behavior than performing by herself, because indifferent points in Fig.1 are under an influence of both impulsivity and inconsistency which should be dissociated by utilizing the q-exponential discount function.

As noted above, we have also fitted the conventional exponential and hyperbolic discount functions (equation 1 and 2) which are not capable of 
distinguishing imconsistency from impulsivity, to the group median data, in addition to the q-exponential discount function. The fitted curves are presented in Figure 2. The estimated parameter values are presented in Table 1. As can be seen from Table 1, participants were more inconsistent (smaller $q$ ) in making an intertemporal decision for someone else than for oneself. Furthermore, impulsivity parameter $k_{q}$ in the q-exponential discount function is larger for someone else than for oneself; in contrast, discount parameters in the exponential and hyperbolic discounting $\left(k_{e}\right.$ and $\left.k_{h}\right)$ were smaller for someone else than for oneself, indicating that conventional (simple) exponential and hyperbolic discount functions could not distinguish the influence of impulsivity from inconsistency in intertemporal choice, as predicted in the above analysis on the property of the q-exponential discounting (Section 2), first conducted in the present study. Namely, seemingly patient intertemporal choice for someone else in the distant future (>1 year) (Fig. 1) may be due to inconsistency (a decline in discount rates as time passes, "decreasing impatience") and patience in the planning of future intertemporal choice, rather than real patience in the actual intertemporal actions at $\mathrm{D}=0$ (which is represented as small $k_{q}$ values in the q-exponential discount function).

Moreover, as we have seen in Section 2, although it has originally been proposed that q parameter is defined in $[0,1)[10]$ and we followed this proposed constraints in our previous study [11], the definition domain of q should be extended to $\mathrm{q}<0$, because when our nonlinear curve fitting was conducted under the constraint that $\mathrm{q}$ is non-negative, estimated values of the $\mathrm{q}$ parameter in both oneself and someone else-versions of q-exponential discount functions were 0 , indicating a poor resolution of consistency between the two conditions. Nevertheless, it should be noted that there was no evidence that decision by other person, instead of a decision-maker herself, mitigates irrationality in intertemporal choice, even when $\mathrm{q}$ is constrained not to be negative.

\section{Conclusions and implications for economic policy-making}

Our empirical estimation of q-parameter in q-exponential discount function in intertemporal choice for oneself versus someone else shows that (i) irrationality in intertemporal choice is even stronger when the outcome is irrelevant to the decision-maker oneself, and (ii) it should be allowed for q-parameter to be negative when the q-exponential function is utilized in intertemporal choice studies. Future studies should examine neuropsychological processes underlying the observed exacerbation of irrationality in intertemporal choice when decision-makers are not involved in the outcome. 
In neuroeconomics, a currently dominant view on the dynamic inconsistency in temporal discounting [13] states the reason for the inconsistency might be that immediate rewards induce affective (or even "visceral") responses of subjects because of its saliency, leading to impulsive choice when the rewards are in the near future. This account, however, may not be true when the outcome is irrelevant to a decision-maker herself. Also, it is apparent that in governmental economic policy-making, the outcomes are not strongly relevant to decision-makers themselves but strongly relevant to other people in the society. Therefore, there is a controversy regarding the effectiveness of paternalistic policy by governmental officials for reducing irrational behavior in individuals [14-15]. Future studies should examine how the irrationality in intertemporal choice for others can be mitigated. This may also hold when the intertemporal choice is over generations such as policy-making against global warming.

\section{Limitations and future study directions}

Because we employed only graduate students in the present study, future studies should examine whether the observed discrepancy in impulsivity and inconsistency in intertemporal choice between for oneself and someone else can be extended to other sociologically different populations (e.g. children, elderly people) and neuropsychiatrically distinct populations (e.g. heroin addicts, attention deficit hyperactivity disorder patients), with larger sample sizes. This point is critical for determining whether the present findings are directly applicable to economic policy-making processes. Before confirming that the present observations can be generalized to many types/classes of people in the societies, a simple application of the present findings to real economic policies should be avoided. The present study has provided a basis for future studies towards this direction with the Tsallis' statistics based q-exponential discount function. It is to be noted that conventional discount functions such as exponential and hyperbolic models, which do not have a parameter of inconsistency, cannot be utilized for these objectives.

Furthermore, our previous studies indicated that temporal processing in the brain may play pivotal roles in determining functional types of temporal discounting $[3,7]$. Therefore, future studies should explore the utilities of statistical physics-based human temporal processing models [16-18] in the investigations into intertemporal choice, for a better understanding of human temporal discounting and a development of statistical physics. 


\section{References}

[1] Ainslie G.. Precis of Breakdown of Will. Behav Brain Sci. 2005 28: 635-650

[2] Frederick, S., Loewenstein, G., \& O’Donoghue, T. Time discounting and time preference: A critical review. Journal of Economic Literature, 40, (2002)351-401.

[3] Takahashi T. Loss of self-control in intertemporal choice may be attributable to logarithmic time-perception. Med Hypotheses. 2005;65(4):691-693.

[4] Ohmura Y, Takahashi T, Kitamura N. Discounting delayed and probabilistic monetary gains and losses by smokers of cigarettes. Psychopharmacology (Berl). 2005 182:508-515.

[5] Takahashi, T. Cortisol levels and time-discounting of monetary gain in humans. Neuroreport, 15 (2004) 2145-2147.

[6] Takahashi T, Sakaguchi K, Oki M, Homma S, Hasegawa T. Testosterone levels and discounting delayed monetary gains and losses in male humans. NeuroEndocrinol Lett. 27(4):439-444 2006

[7] Takahashi T. Time-estimation error following Weber-Fechner law may explain subadditive time-discounting. Med Hypotheses. 2006;67(6):1372-1374.

[8] Motonishi T. Dynamic inconsistency in the public supply of liquidity. Japanese Economic Review 55 (4): 401-417 2004

[9] Lee D. Neuroeconomics: making risky choices in the brain. Nat Neurosci. 2005 8(9):1129-1130.

[10] Cajueiro D.O. A note on the relevance of the q-exponential function in the context of intertemporal choices. Physica A 364 (2006) 385-388.

[11] Takahashi T, Oono H, and Radford MHB, Empirical estimation of consistency parameter in intertemporal choice based on Tsallis' statistics. Physica A 381 338-342 (2007) 
[12] Takahashi T, Oono H, Radford MHB. Comparison of probabilistic choice models in humans. Behavioral and Brain Functions 2007, 3:20

[13] McClure SM, Laibson DI, Loewenstein G, Cohen JD. Separate neural systems value immediate and delayed monetary rewards. Science. 2004 306(5695):503-507.

[14] Mitchell G. Libertarian paternalism is an oxymoron. Northwestern University Law Review 99 (3): 1245-1277

[15] Sunstein CR, Thaler RH Libertarian paternalism is not an oxymoron. University of Chicago Law Review 70 (4): 1159-1202 2003

[16] Johansen, A "Response times of internauts", Physica A 296(3-4) 539-546 (2001).

[17] Johansen, A "Probing human response times", Physica A 338 (1-2): 286-291 (2004).

[18] Chessa A. G, and Murre, J.M.J. "A memory model for internet hits after media exposure". Physica A 333: 541-552 (2004) 
Table 1. Parameters in intertemproal choice for oneself and someone else

\begin{tabular}{lllll} 
& Oneself & & \multicolumn{2}{c}{ Someone else (Other) } \\
\hline$q$-exponential & $q$ & $k_{q}$ & $q$ & $k_{q}$ \\
& -2.629810 & 0.00896 & -8.8925 & 0.07032 \\
& & & & $k_{e}$ \\
\hline Exponential & & $k_{e}$ & & 0.0007083 \\
\hline Hyperbolic & 0.00149 & $k_{h}$ \\
& & $k_{h}$ & 0.0014942 \\
\hline
\end{tabular}

Consistency in intertemporal choice (q parameter in q-exponential discount function) is larger for oneself than for someone else. This indicates that intertemporal choice behavior for someone else is more inconsistent than for oneself. Impulsivity parameters in exponential and hyperbolic discounting $\left(k_{e}\right.$ and $k_{h}$ ) are larger for oneself; however, impulsivity parameter in q-exponential discount function $\left(k_{q}\right)$ is smaller for self-relevant intertemporal choice. These data imply that when impulsivity and inconsistency are dissociated, an intertemporal choice action for someone else is more impulsive and inconsistent than for oneself. Fitted curves with these estimated parameters are presented in Fig.2. 


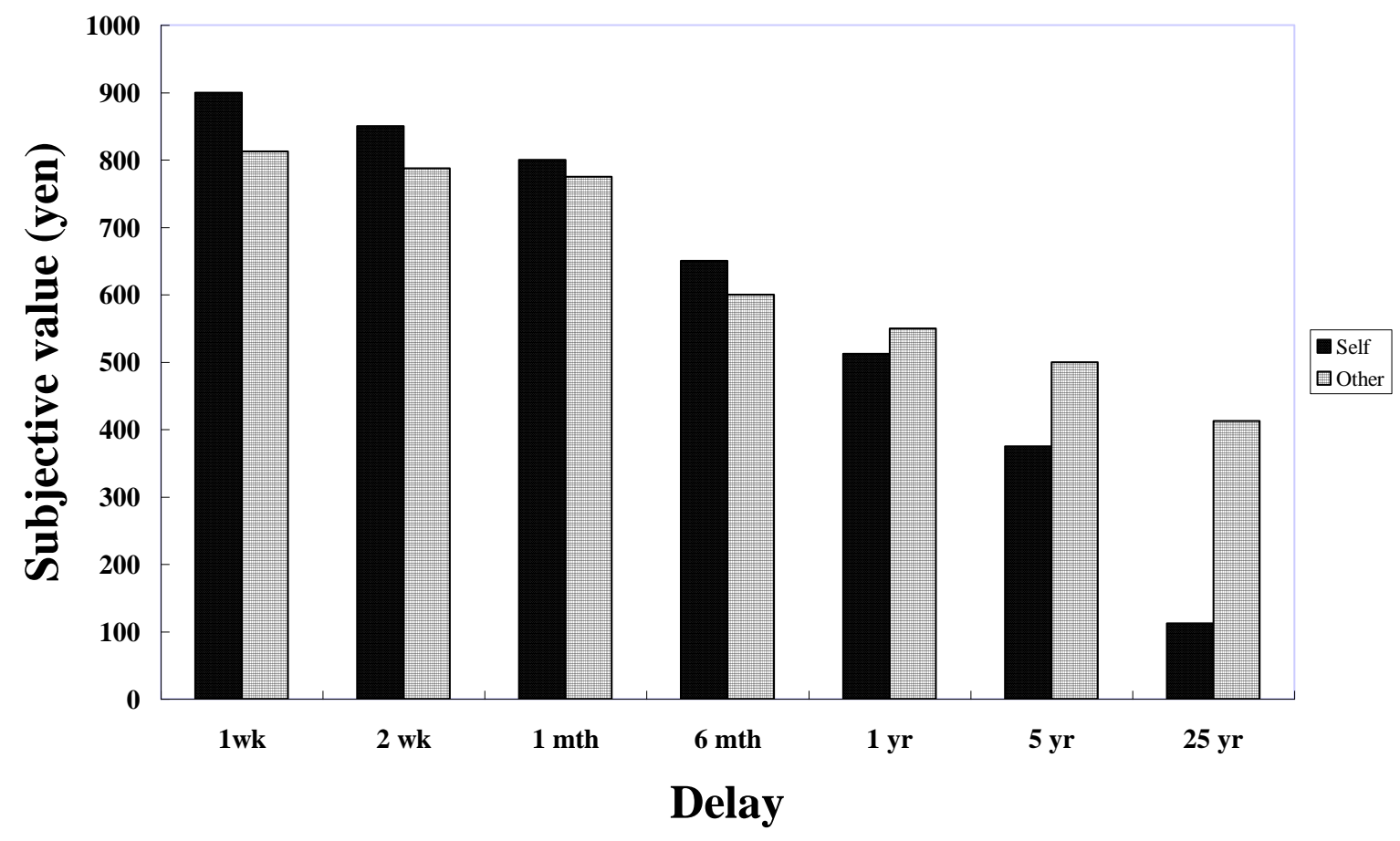

Fig. 1

Group median data of discounted present values of delayed monetary rewards for oneself (Self) and someone else (Other) at each delay. In the distant future (delay>1 year), the discounted present values of delayed rewards were larger in intertemporal choice for someone else than for oneself. 
Fig. 2

Fitting of $q$-exponential (solid curve), hyperbolic (dashed curve), and exponential (dotted curve) models for group data of intertemporal choice for (a) oneself (Self, blue points) and (b) someone else (Other, red points). The estimated parameters for each model are presented in Table 1. Order of goodness-of-fit was [q-exponential>hyperbolic>exponential] for both (a) and (b) (see Section 2).
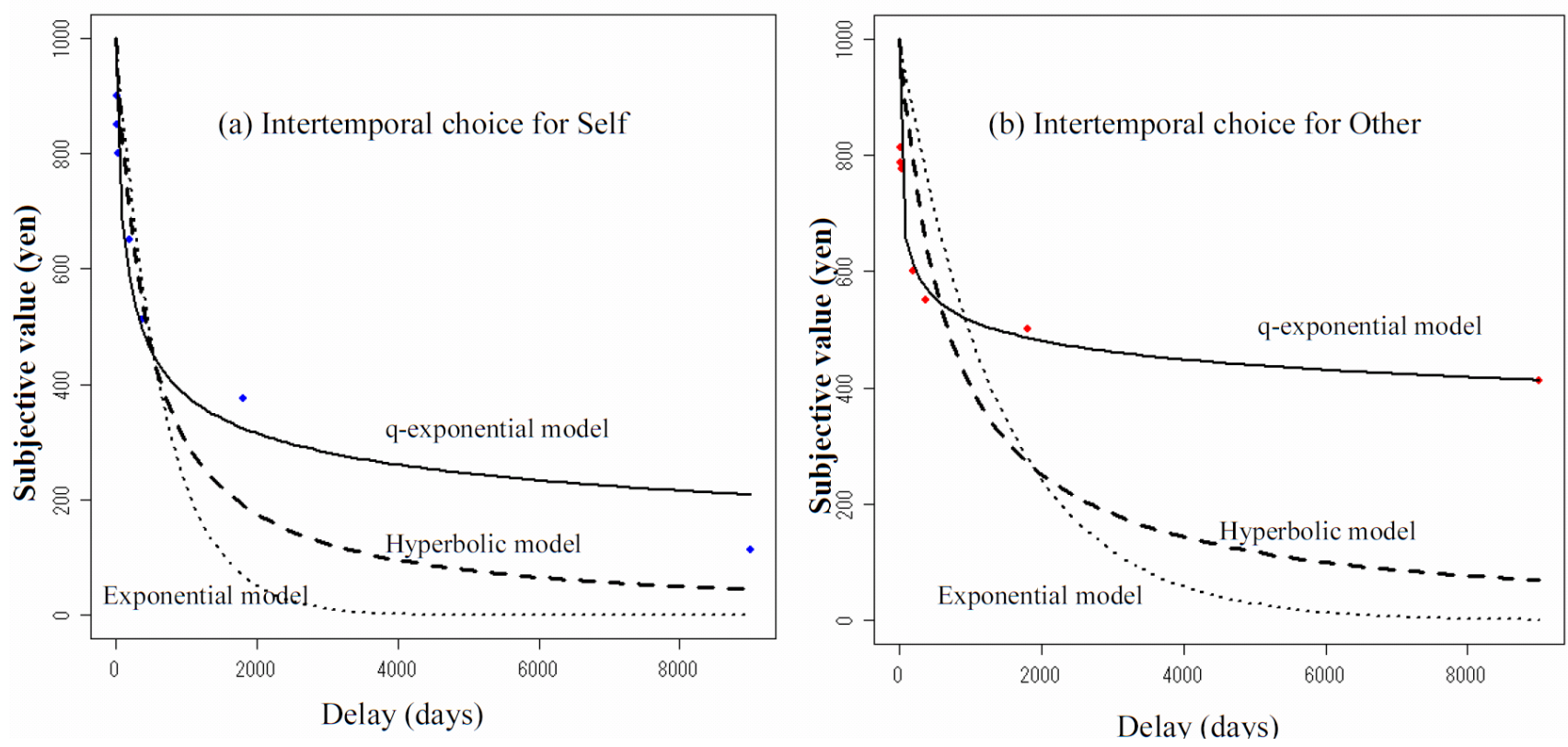